\title{
An evolvable space telescope for future astronomical missions 2015 update
}

Ronald S. Polidan, James B. Breckinridge, Charles F. Lillie, Howard A. MacEwen, Martin R. Flannery, et al.

Ronald S. Polidan, James B. Breckinridge, Charles F. Lillie, Howard A. MacEwen, Martin R. Flannery, Dean R. Dailey, Brian Baldauf, David Makowski, Gerald L. Rafanelli, "An evolvable space telescope for future astronomical missions 2015 update," Proc. SPIE 9602, UV/Optical/IR Space Telescopes and Instruments: Innovative Technologies and Concepts VII, 960207 (22 September 2015); doi: 10.1117/12.2189105

Event: SPIE Optical Engineering + Applications, 2015, San Diego, California, United States 


\title{
An Evolvable Space Telescope for Future Astronomical Missions 2015 Update
}

\author{
Ronald S Polidan $^{* a}$ James B Breckinridge $^{\mathrm{b}}$, Charles F Lillie ${ }^{\mathrm{c}}$, Howard A MacEwen ${ }^{\mathrm{d}}$, \\ Martin R Flannery ${ }^{\mathrm{a}}$, Dean R Dailey ${ }^{\mathrm{a}}$, Brian Baldauf ${ }^{\mathrm{a}}$, David Makowski ${ }^{\mathrm{a}}$, and Gerard L Rafanelli ${ }^{\mathrm{e}}$ \\ ${ }^{a}$ Northrop Grumman Aerospace Systems, One Space Park Dr., Redondo Beach, CA 90278 \\ ${ }^{\mathrm{b}}$ Breckinridge Associates, LLC, 985 East California Boulevard, Pasadena, CA. 91106 \\ ${ }^{c}$ Lillie Consulting LLC, 6202 Vista del Mar, Playa del Rey, CA 90293 \\ ${ }^{\mathrm{d}}$ Reviresco LLC, 4901 Loosestrife Court, Annandale, VA 22003 \\ ${ }^{\mathrm{e}}$ Raytheon Space and Airborne Systems, 2000 E. El Segundo Blvd, El Segundo, CA 90245
}

\begin{abstract}
In 2014 we presented a concept for an Evolvable Space Telescope (EST) that was assembled on orbit in 3 stages, growing from a 4x12 meter telescope in Stage 1, to a 12-meter filled aperture in Stage 2, and then to a 20-meter filled aperture in Stage 3. Stage 1 is launched as a fully functional telescope and begins gathering science data immediately after checkout on orbit. This observatory is then periodically augmented in space with additional mirror segments, structures, and newer instruments to evolve the telescope over the years to a 20-meter space telescope. In this 2015 update of EST we focus upon three items: 1) a restructured Stage 1 EST with three mirror segments forming an off-axis telescope (half a 12-meter filled aperture); 2) more details on the value and architecture of the prime focus instrument accommodation; and 3) a more in depth discussion of the essential in-space infrastructure, early ground testing and a concept for an International Space Station testbed called MoDEST. In addition to the EST discussions we introduce a different alternative telescope architecture: a Rotating Synthetic Aperture (RSA). This is a rectangular primary mirror that can be rotated to fill the UV-plane. The original concept was developed by Raytheon Space and Airborne Systems for non-astronomical applications. In collaboration with Raytheon we have begun to explore the RSA approach as an astronomical space telescope and have initiated studies of science and cost performance.
\end{abstract}

Keywords: Space telescopes, alternate architectures, segmented telescopes, in-space assembly and servicing, synthetic apertures

\section{INTRODUCTION}

The scientific case for very large telescopes has been very well established by various studies over the past decade, most recently and particularly well by the Association of Universities for Research in Astronomy (AURA) "From Cosmic Birth to Living Earths" study ${ }^{1}$ in which they present and discuss a concept for a High Definition Space Telescope (HDST). Building this observatory in the current and likely future cost constrained environment will be a challenge. Space telescope costing studies, for example Stahl et $\mathrm{al}^{2}$, have developed empirical relationships that indicate that cost of large astronomical telescopes will greatly challenge the existing NASA Astrophysics budget. Telescope, spacecraft, and instrument technology has been advancing at a rapid pace, enabling new approaches to building and operating future large observatories. We as a nation are beginning to expand our in-space infrastructure, further enhancing our options for large space telescopes. In going forward we must examine alternate telescope architectures that reduce cost, shorten schedules, expand performance, and take advantage of new or pending technology and the developing space infrastructure and not be locked into traditional approaches. While there are certainly technical challenges with any new

*ron.polidan@ngc.com; phone 1 310-812-0199; fax 1 310-812-7443; northropgrumman.com

UV/Optical/IR Space Telescopes and Instruments: Innovative Technologies and Concepts VII, edited by Howard A. MacEwen, James B. Breckinridge, Proc. of SPIE Vol. 9602, 960207 (c) 2015 SPIE · CCC code: 0277-786X/15/\$18 - doi: 10.1117/12.2189105 
architecture, the greater challenge is more often the culture changes that are required to abandon the comfortable traditional approach and adopt a new path. In this paper we look at two alternate architectures for future large astronomical observatories. An Evolvable Space Telescope (EST) concept that builds the observatory in stages in space utilizing our in-space servicing and assembly infrastructure to keep annual costs relatively low while enabling science return early in the program and producing a very long lived observatory that evolves in both size and science capability to take advantage of the advancing technology and adapting to new science questions as they arise. The EST concept was first presented at SPIE in 2014 (Polidan et $\mathrm{al}^{3}$ ). The second large telescope architecture is a Rotating Synthetic Aperture (RSA) concept that utilizes a large rectangular aperture and rotates to fill the UV-plane. This concept was initially developed by Raytheon Space and Airborne Systems for non-NASA applications and is being reexamined for application to astrophysics by a combined Northrop/Raytheon team. RSA offers potential cost and complexity reductions while delivering exceptional science performance.

\section{EST SCIENCE GOALS}

The EST science goals will only very briefly be discussed in this paper, since the science goals for future large observatories are outlined in great detail in the AURA "From Cosmic Birth to Living Earths" study". The three top-level EST primary science goals will likely be:

- Characterize exoplanets and, ideally, discover signatures of life (specifically, spectral biomarkers) for a statistically significant number of suspected planets outside the solar system.

- Expand our understanding of the origin and evolution of the universe, including star and planet formation and galaxy assembly.

- Further explore the distribution and the nature of dark energy and dark matter.

Of course there is a wide range of additional science that would be enabled by EST. Stage 1 in particular is designed to be an exceptional UV astrophysics observatory with its large collecting area and high efficiency prime focus instruments. However it is the three top level science goals that carry the most weight in the setting of top level requirements for EST performance. These top level requirements are enumerated in Table 1.

Table 1: EST Design Reference Concept Top Level Requirements

\begin{tabular}{|l|c|c|l|}
\hline \multicolumn{1}{|c|}{ Parameter } & Requirement & Goal & \multicolumn{1}{|c|}{ Notes } \\
\hline Telescope Aperture & $>10 \mathrm{~m}$ & $>16 \mathrm{~m}$ & $\sim$ AURA HDST concept \\
\hline Stage 1 & Off-axis, 3 segment & $4 \times 12 \mathrm{~m}$ & Three hexagonal segments \\
\hline Stage 2 & Filled Aperture & $12 \mathrm{~m}$ & Six hexagonal segments \\
\hline Stage 3 & Filled Aperture & $20 \mathrm{~m}$ & Eighteen hexagonal segments \\
\hline Wavelength & $100-2400 \mathrm{~nm}$ & $90-8000 \mathrm{~nm}$ & UVOIR, MIR under evaluation \\
\hline Field of View & 5 to $8 \mathrm{arcmin}$ & $40 \mathrm{arcmin}$ & Wide field VNIR imaging \\
\hline Diffraction Limit & $500 \mathrm{~nm}$ & $250 \mathrm{~nm}$ & Enhanced UV/Optical resolution \\
\hline Primary Segment Size & $2.4 \mathrm{~m}$ & $3.93 \mathrm{~m}$ & flat to flat \\
\hline Primary Mirror Temp & $<200 \mathrm{~K}$ & $150 \mathrm{~K}$ & Minimize heater power \\
\hline Design Lifetime & 15 years & $>30$ years & On-orbit assembly and servicing \\
\hline
\end{tabular}




\section{EST CONCEPT SUMMARY}

In 2014 (Polidan et $\mathrm{al}^{3}$ ) we presented the basic Evolvable Space Telescope concept. The concept had three major measures of merit, most notably in terms of performance and affordability. Three that particularly distinguish the EST mission concept from other approaches are: evolvability, adaptability, and serviceability.

- Evolvability. This, of course, is the core of the EST concept, and directly implies that there will be several configurations of EST as it evolves in several "Stages" over the observatory's lifetime. These Stages will be separated from each other by several years (nominally five) and will provide substantial performance advances based upon the evolution of the science drivers and/or available technologies. The Stage 1 EST is launched as a fully functional observatory. Subsequent Stages would increase the telescope collecting area, add new instruments and/or upgrade telescope or spacecraft subsystems. Stage 1 will be designed to provide significant capability for current science drivers and will form the core of the future larger telescopes (i.e., starting with the collecting area of an equivalent $\sim 7$-meter telescope and evolving to a 16 to 20 meter aperture telescope).

- Adaptability. Evolvability will not occur in a static environment, and EST will have to respond to unplanned changes in multiple conditions. Many are quite obvious, and the following list in no way exhausts the possibilities:

- Budgetary changes, either negative or positive, affecting either the entire NASA budget or priorities within that budget.

- Political changes, either within the nation or geopolitical in nature.

- Technical failures or shortcomings (or, alternatively, unexpected technical successes).

- Serviceability. This measure is essential to each of the preceding two, since it directly addresses the program's ability to maintain performance against failures or wear and tear, major or minor; and the ability to enhance or upgrade systems, again including minor enhancements or Stage changes. It is directly related to the continued performance of EST over many decades, lasting perhaps as long as 50 or more years with periodic robotic and/or crewed servicing.

The baseline EST is designed to be a Sun-Earth $\mathrm{L}_{2}$ (SEL2) observatory. It begins with an initial development and launch of a "core" fully functional telescope with instruments. Specifically, this first Stage is a three segment off axis telescope (in 2014 Stage 1 was envisioned as a two element "bow-tie" arrangement). Subsequently, this observatory is augmented with additional mirror segments, new instruments, spacecraft subsystem replacements/upgrades, and other needed infrastructure elements to build a filled aperture telescope. Follow-on augmentations would add additional mirror segments and instruments along with any needed spacecraft and infrastructure replacements/upgrades to build an even larger filled aperture observatory.

For simplicity for this study we have adopted a fixed mirror element size of $\sim 4$ meters flat-to-flat, or approximately 4.5 meters point-to-point, although these dimensions are purely illustrative. There is nothing in the architecture that requires a specific size for each mirror element and each mirror element can be a single mirror segment (the baseline for this study) or composed of smaller mirror segments that are built up into the basic mirror element. The EST approach can accommodate any segment size, with the telescope size and total collecting scaling with the mirror element size, but the EST architecture functions equally well with smaller or larger mirror segments.

This evolution of the EST Primary Mirror Assembly (PMA) is described below and shown pictorially in Figure 1. Illustrations of the full observatory are presented in Figure 2.

- EST Stage 1 is indicated in Figure 1 by the three grey hexagons and the central circle (changed from the 2014 two-element "bow-tie" Stage 1). The hexagons form the initial PMA, and will typically be formed to the master prescription. The central circle represents the secondary mirror, and will be designed to serve the same role throughout Stages 1, 2, and 3. These elements will all be orbited and assembled using the first EST launch, providing an off-axis aperture on the order of $12 \mathrm{~m}$ by $4.5 \mathrm{~m}$ with a prime focus instrument suite.

- Stage 2. At the chosen time (about half a decade following Stage 1), three additional segments (shown in dark blue) will be launched, along with other components (e.g., new instruments and a new tensegrity truss structure) to form the Stage 2 EST: a $12 \mathrm{~m}$ filled aperture, with a prime focus and/or a Cassegrain configuration.

- Stage 3. Again, approximately a half-decade following the preceding launch, the next stage of telescope evolution will occur, in this case adding 12 mirror segments (in green) to form a $20 \mathrm{~m}$ aperture for the telescope, in a Cassegrain configuration. In this case, since the largest dimension of the PMA will be significantly 
increased, the upgrade will include at least a new sunshield, and tensegrity truss and other replacement components and servicing as needed.

- Stage $4+$. Later stages of EST remain possibilities for examination in the future.

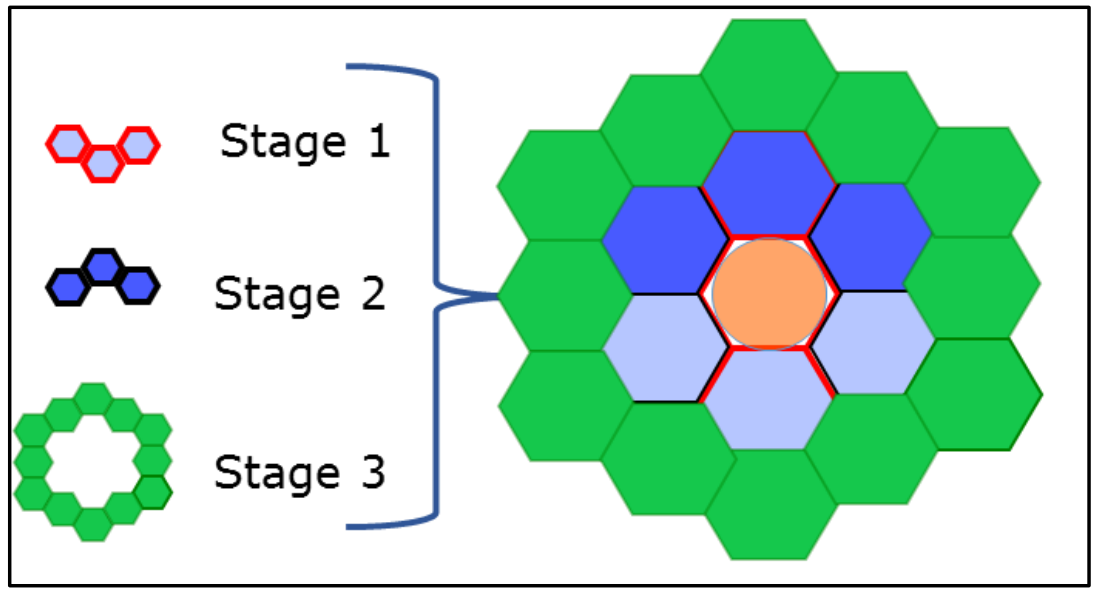

Figure 1: Pictorial configuration for EST Stages 1, 2, and 3

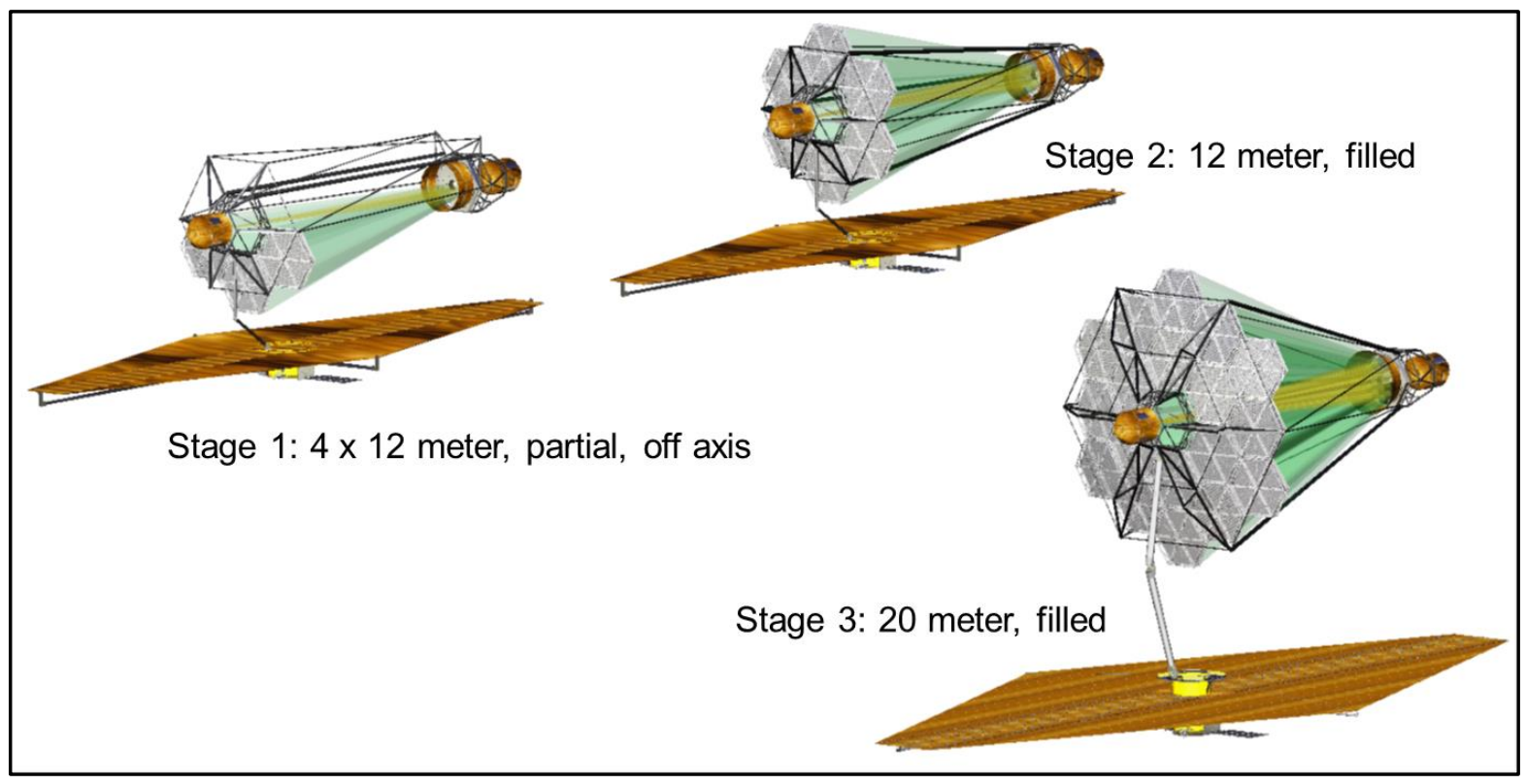

Figure 2: Representations of the full EST observatory Stages 1, 2, and 3 


\section{2015 EST DEVELOPMENTS}

In 2015 we continued the development of EST with three primary objectives: 1) to revisit the 2014 Stage 1 approach and architecture vis-à-vis mission science goals and the overarching (3 stage) system design; 2) to explore more thoroughly the prime focus instrument accommodation; and 3) to examine the existing and planned in-space assembly and servicing infrastructure and its application to EST.

\subsection{Reassessment of EST Stage 1}

At the end of the 2014 study it was realized that the "bow-tie" EST Stage 1 had less collecting area than desired to meet science goals and that the point spread function from the "bow-tie" was less than optimal. Eventually, a 3 adjacent mirror element architecture was settled upon as the preferred option (Figures 1 and 2). This increased the collecting area by $50 \%$ over the bow-tie version and gave a vastly improved point spread function. This change necessitated a modification of the telescope structure which also improved performance by moving EST Stage 1 from an on-axis obscured system to an off axis, unobscured system. It also simplified the transition to EST Stage 2, evened out the fabrication schedule, and made better use of the lifting capacity of the rockets used for the first two stages. The "bowtie" configuration, with its separated elements, made demands on the telescope alignment system that the contiguous half-aperture adjacent segments do not. In addition, looking at both coronagraphic and UV spectroscopy instruments for Stage 1, the prime-focus instrument package was both more practical and provided higher performance than was possible with the "bow-tie" configuration.

\subsection{EST Optical System Performance}

\subsubsection{Introduction}

The EST concept is versatile and flexible to respond to the continuously evolving needs of a diverse astronomical community in the presence of budgetary and technical constraints. By launching the telescope in parts and assembling it in space we have the opportunity to replace aging hardware with more capable hardware without the expense and waste of discarding the telescope and its instruments as will happen to HST and JWST. Preserving our investment in on-orbit hardware will lead to affordable observatories. EST's primary mirror is segmented. The concept discussed above is to robotically add to or replace segments as needed, in a manner similar to that used by the AAReST ${ }^{4}$ project at Caltech. The HST has been very successful in large part because of our ability to swap out instruments. But there is no reason that, if we design the system properly we cannot also swap out telescope optical elements to optimize the configuration in-space to address the then current priorities of the astronomical community. Note that because of the rotational symmetry of Stage 1 about the optical axis, the non-recurring engineering costs of Stage 1 are fully applicable to Stage 2. In fact, one can build two identical Stage 1 mirror assemblies, launch the Stage 1 tri-hex system and observe with this off-axis telescope. Then, when sufficient funds are available launch the second unit, rotate it around, and match it to the Stage 1 system to create the 12-meter Stage 2 on-axis, filled aperture telescope. Figure 3 below shows a concept for the EST primary mirror and metering structure. Light, shown as a broken line enters the system from right to left, reflects from the concave primary mirror and converges to a focus near the right end of the metering structure. At the right end of the metering structure is a flange for docking either the Cassegrain secondary or a prime focus instrument assembly (PFIA). 


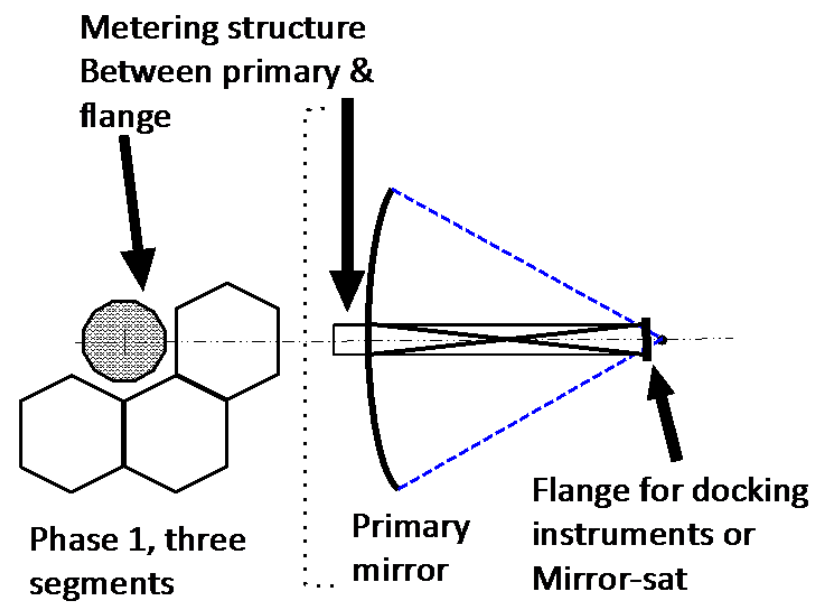

Figure 3: The Evolvable Space Telescope core structure. The broken line shows light reflected from the primary mirror converging to a focus near the flange for docking instruments or mirrors. The metering structure shown is notional. The three hex segments of the primary mirror are used in an unobscured telescope.

\subsubsection{Classical Cassegrain}

Figure 4 below shows a concept for the EST with a classical Cassegrain secondary mirror assembly package docked to the right end of the metering structure shown in Figure 3. The secondary mirror assembly can be undocked and removed in space and replaced with other modules such as a prime-focus instrument assembly or another secondary mirror with an optical figure optimized for a three mirror anastigmat (TMA) wide-field system depending on the science measurement priorities. If the instruments at the Cassegrain focus contain diffraction gratings and fold mirrors that produce Fresnel polarization aberrations, several polarization mitigation approaches are available ${ }^{5,6}$.

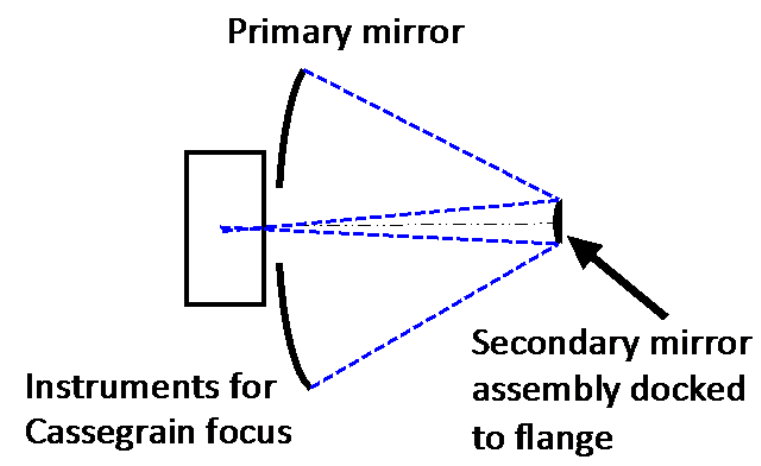

Figure 4: A concept for the EST with a classical Cassegrain mirror assembly package docked to the right end of the metering structure shown in Figure 3.

\subsubsection{Prime Focus}

In this section, we introduce the concept of an EST prime focus and examine the performance of a prime focus spacetelescope configuration. An instrument at prime focus enables us to minimize scattered light, eliminate a structurally sensitive optical element (the secondary), minimize polarization aberrations, reduce thermal disturbances and maximize transmittance for observing extremely faint objects and for observational UV astronomy.

For many years, before the dawn of the age of space telescopes, ground-based astronomers used the Cassegrain configuration for their telescopes. This design approach was chosen to minimize telescope dome structures and place the instruments behind the primary mirror where there was a large volume available. The mass of the instrument was also 
near the heavy primary mirror and easy to reach by an astronomer peering through the telescope eyepiece. Large telescopes such as the 5-meter Hale telescope and the 3-meter Shane telescope were built with prime focus "cages" where the observer rode along with the science instrument. These cages are less valuable today because mass and volume are constrained. Although several ground based telescopes do have wide field cameras at their prime focus to maximize their sensitivity for faint objects. For space telescopes, the HST is a classical Cassegrain and the JWST is a TMA whose primary and secondary are in classical Cassegrain configuration. Both inherit this tradition from groundbased astronomy. Ground telescopes are constrained to operate at atmospheric temperature, within the Earth's atmosphere and gravitational field. However, for space telescopes some of these ground-based constraints are missing and a prime focus system may be less expensive to build, and refurbish for a given science return than the Cassegrain. The cost of a telescope depends to a large extent on the number of large optical elements that need to be controlled to nanometer tolerances. The EST gives us an opportunity to conduct trades and analyses and explore the possibility of a new more cost effective space telescope optical configuration.

Figure 5 is a sketch of a prime focus telescope system designed for wide-field and coronagraphic imaging by the Stage 1 and 2 twelve-meter EST. Light passes from the right to the left to reflect from the $12 \mathrm{~m} \mathrm{~F} / \#=2.5 \mathrm{EST}$ primary mirror. The box shown just to the right of the prime focus is the prime focus instrument assembly (PFIA) whose diameter is 3 to 4 meters and length a few meters to hold prime focus instruments such as UV spectrometers, imagers, coronagraphs, wide-field cameras, etc. The focal plane is shared (like HST and JWST) with several instruments contained within the PFIA. A metering structure (see Figure 3 above) separates the primary mirror from the prime focus. A free-flying spacecraft can be attached to the PFIA for unlatching and precision docking of the PFIA from the end of the metering structure for replacement of the entire instrument assembly. Following one of the HST system architecture features, the EST is designed for instrument and mirror upgrades based on new detector developments, new, more efficient optical designs and new, evolving science measurement objectives during the 30 -year anticipated lifetime of the primary mirror assembly.

We have selected one example instrument, an imaging system, for discussion here. The prime focus is $30 \mathrm{~m}$ from the vertex of the primary. Light passes through a field lens and expands to fill the primary of an inverse Schwarzschild ${ }^{7,8}$ reimaging system mounted just beyond prime focus. The field lens relays an image of each segment of the primary onto the 0.6-meter diameter active segmented number one mirror of the Schwarzschild. Primary mirror wavefront errors (tilt, piston and surface) caused by fabrication and time-dependent thermal, dynamics and structural effects are compensated for at the $60-\mathrm{cm}$ segmented secondary mirror. Light reflects from the secondary to the convex tertiary and then to the focal plane indicated by the circle or to a dispersive element in the case of a spectrometer. The convex curvature on the tertiary combines with the concave powered mirrors 1 and 2 to provide a flat field at the focus. Adjusting the design of the Schwarzschild relay controls the detector sampling frequency. For small fields of view such as those needed for exoplanet coronagraphy and high contrast imaging of stellar neighborhoods, the transparent field lens can be swung out of the way and possibly replaced by a diffractive mask for unprecedented direct control of the unwanted radiation after the first reflection. There are only three reflecting optical surfaces before the detector. There are no fold mirrors in the system, which will minimize polarization aberrations and their deleterious effect on the PSF $^{9,10,11}$.

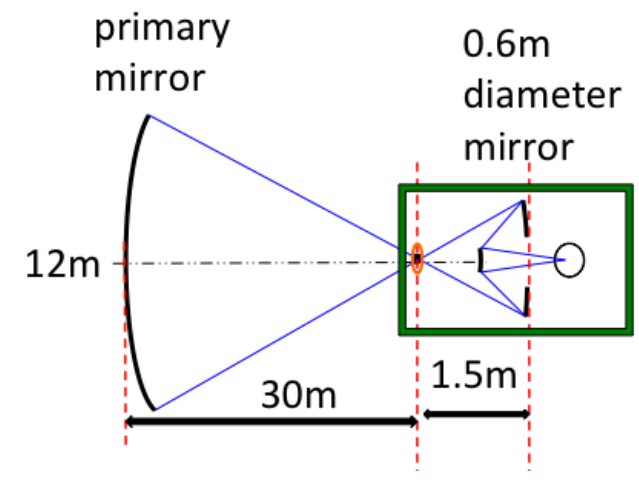

Figure 5: Shown is a prime focus concept for the EST system. A prime-focus instrument assembly (PFIA) is shown docked to the flange at the right end of the metering structure, $30 \mathrm{~m}$ from the vertex of the primary mirror that separates the primary from the PFIA. The metering structure (not shown here) separates the primary mirror from the flange (not shown). 


\subsubsection{Point spread function}

The PSF for the prime focus system is shown in Figure 6. As discussed in Section 3, the first Stage of the EST will use three 4-m class segments oriented as shown in Figure 1. Figure 6 shows the point-spread-function (PSF) for the threehex pupil for 4-m flat-to-flat segments with 5-mm gaps. Geometric aberrations are fully corrected. Polarization aberrations were not calculated, but should be minimal or easily mitigated since the basic three-mirror system has no flat fold elements to add significant Fresnel polarization aberrations. On the left is a $\log _{10}$ intensity image of the PSF with a Field of view of $4 \times 4$ arc seconds. On the right is a cross section through the PSF taken at 30 degrees as shown by the solid white line. The horizontal axis is the FOV from -.5 to +.5 arc seconds and the vertical axis is $\log _{10}$ intensity from 0 to $10^{-7}$. With an effective light gathering area of $\sim 40-\mathrm{m}^{2}$ and a maximum base line of 12-meters, this Stage 1 system will be a very capable and productive first stage for EST. Redesign of the instruments, in particular the location and shape of the telescope pupil will be necessary for the 12-meter 6-Hex Stage 2 and the eventual 20-meter 18-Hex Stage 3.

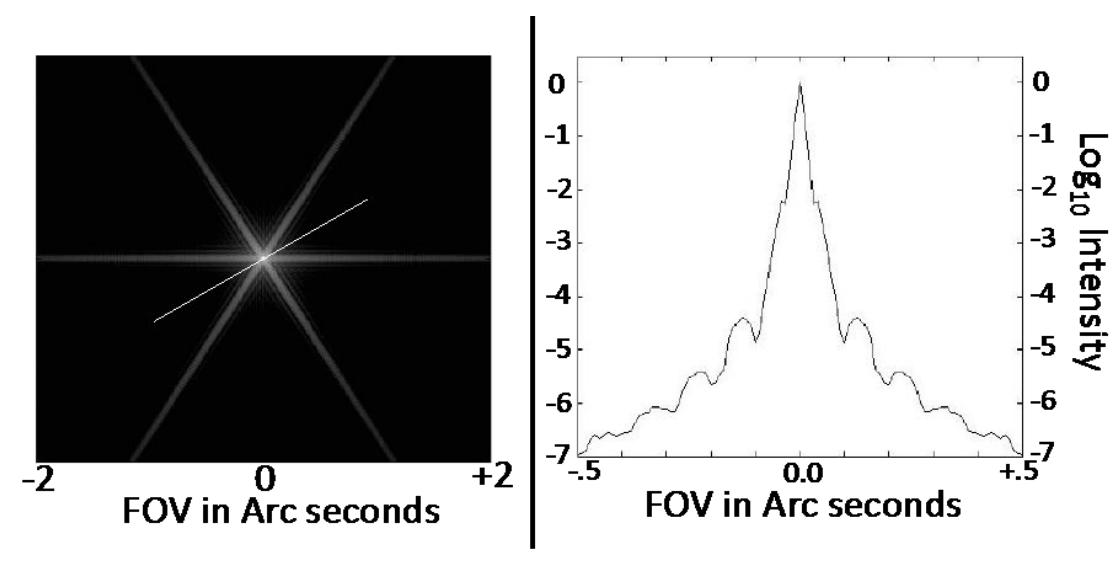

Figure 6: The point-spread-function (PSF) for the three-hex pupil with 4-meter flat to flat segments and 5-mm gaps. Geometric aberrations are fully corrected. Polarization aberrations were not calculated, but should be minimum or easily mitigated since the basic three-mirror system has no flat fold elements to add significant Fresnel polarization aberrations. On the left is a Log $_{10}$ intensity image of the PSF with a Field of view of $4 \times 4$ arc seconds. On the right is a cross section through the PSF taken at 30 degrees as shown by the solid white line. The horizontal axis is the FOV from - .5 to +.5 arc seconds and the vertical axis is $\log _{10}$ intensity from 0 to $10^{-7}$.

The advantages of the EST and the PFIA system shown in Figure 5 are:

1. There are only three reflections from powered optical elements and no reflections from fold mirrors before the focal plane. This will minimize reflection losses and minimize system Fresnel polarization aberrations and maximize transmittance for imaging objects at the threshold of detection and UV spectroscopy and imaging.

2. For narrow field ( $\sim 10$ arc second) UV imaging and spectroscopy the inverse Schwarzschild optics can be replaced by a convex grating and used with and without transmissive corrective optics to maximize sensitivity in the 90 to $350 \mathrm{~nm}$ spectral region.

3. For exoplanet coronagraphy, since the polarization aberrations are minimal there will probably be insignificant difference between the aberrations in the two orthogonal polarizations and no need to polarization-divide the pupil-image for optimal adaptive optics correction. This will avoid a 50\% loss in system transmittance.

4. In a classical Cassegrain system such as HST and a TMA such as JWST the telescope optical path passes twice along the metering structure separating the primary and secondary. Length errors caused by thermal changes and structural dynamics of this metering structure are doubled in a Cassegrain configuration compared to a prime focus system.

5. Because the PFIA looks directly at the primary mirror (unlike the Cassegrain which needs to be sky-baffled) scattered light control is much easier and the long baffle that runs from the primary to the secondary in a Cassegrain is not needed, saving mass and eliminating a source of unwanted radiation. 
6. For IR thermal radiators on detectors and optics near the focal plane, the PFIA has a large solid angle to the dark sky, which is advantageous. For Cassegrain telescopes the instrument detector thermal radiator looks at the back end of the warm telescope which is a source of thermal background. Also, for a Cassegrain the back end of the telescope is usually pointed towards the sun or a warm sunshade limiting the solid angle to cold deep space for heat radiation. The prime focus has fewer operational limitations.

7. Unwanted thermal background radiation on the detector from the three-mirror prime focus system is less than that for the typical Cassegrain system which utilizes many reflections, some at steep angles to accommodate spacecraft packaging.

8. Additional resources are needed for more detailed analyses, such as:

a. The diffraction limited FOV achievable with the inverse Schwarzschild with surfaces optimized using modern free-form optics optimization, Without using free-form optics, we have a design that consists of a radiation-hard apochromatic prime-focus corrector with aspherical surfaces plus conical optics in the Inverse Schwarzschild that achieves a diffraction limit at $500 \mathrm{~nm}$ over an 18 -arc sec FOV.

b. Quantitative assessment of polarization aberrations and their effects on coronagraph contrast limits for EST.

c. The Far-UV $(<100 \mathrm{~nm})$ may be accessible using crystal correctors at the prime focus; in which case there is only one reflection (the primary mirror) before a prime-focus UV detector.

d. Advantages of the two-body dynamics of the prime-focus (primary and instruments) compared to the three body (primary, secondary, instruments) dynamics of the Cassegrain.

e. The symmetry of the inverse Schwarzschild may provide a tilt compensation aspect to this design.

\subsubsection{Optical System Summary}

We have shown that the EST optical system is potentially highly capable and responsive to changing requirements and technology and will support a highly diverse set of space astrophysics and exo-planet missions.

\subsection{In-Space Infrastructure}

Essential to the EST concept is the existence of a mature in-space assembly and servicing infrastructure, either purely telerobotic, purely crewed, or most likely a combination of telerobotic and crewed. As stated earlier, leveraging and utilizing in-space assembly and servicing is the key to the success of the Evolvable Space Telescope, particularly given the extended operational lifetime expected of the observatory.

Accomplishing the goals of EST will require a NASA in-space infrastructure capable of:

- Enabling the assembly of a major new astronomical observatory from its earliest stages;

- Readily upgrading to major new capabilities at a fraction of the cost of a new, large space telescope;

- Enough flexibility to incorporate relatively small enhancements to respond to new observational opportunities;

- Maintenance and repairs as necessary, small or large, to maintain a full operational capability despite normal wear and tear or significant anomalies or malfunctions, short of major destruction; and

- Continued observations during transit to and from a servicing location/facility, if only at a (significant) fraction of the full capability.

This, in turn, will require a substantial in-space infrastructure designed for maintenance, upgrading, and repair of large space vehicles. This infrastructure does not yet exist, although many aspects have been examined in various degrees of detail and completeness. A skeleton infrastructure has been created, including the International Space Station (ISS), a number of ground-launched single use logistical spacecraft (Orion, Dragon, Cygnus, ...), and miniature auxiliaries (SPHERES, Cubesats, and the like). The fact that this is clearly inadequate for a robust development of space, both for science and for manned exploration, implies a clear need for a vigorous infrastructure development and deployment. This need has been recognized and thoroughly discussed in the NASA "On-Orbit Satellite Servicing Project Report" 


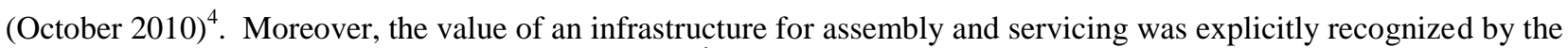
AURA "From Cosmic Birth to Living Earths" report":

"Finally, on-orbit satellite servicing and on-orbit spacecraft assembly are two key technologies that are beyond the scope of any single project, but that could be of great benefit to HDST and many other future missions, were they to be provided. We encourage NASA to consider whether a new on-orbit servicing infrastructure could be developed, perhaps in concert with other agencies or the commercial space sector. Replacement of failed components extended the life of Hubble, and replacement of its instruments greatly enhanced its scientific value. We would want the same for HDST and other missions as well."

The logical starting point would be the development and deployment of two key first generation systems whose capabilities interlock and permit them to operate in coordination with each other. These are an optionally crewable Deep Space Habitat (DSH - effectively a small space station located in cislunar space) and a smaller, unmanned vehicle (termed MiniServ in this paper) that can be based at the DSH and operated in its vicinity or deeper in space for telerobotic assembly and repair missions. Both systems are considered to be well within the current state-of-the-art, although they will obviously benefit from new technology developments should such become available.

Such infrastructure development is the subject of this section, which necessarily assumes certain parameters concerning the telescope itself. The telescope model is, of course, the Evolvable Space Telescope, but many of the same considerations will likely apply to the Rotating Synthetic Aperture (RSA) concept discussed later in this paper and other space telescope architectures. Four assumptions regarding the EST are specifically relevant in the context of that concept:

- The telescope will be designed and built with maintenance/servicing and upgrading/growth specifically in mind. As needed, dedicated service vehicles (potentially developed, fabricated, and operated in conjunction with manned or other programs) will be provided to the telescope program.

- The orbit for the large space telescope will be a Sun-Earth L2 (SEL2) halo orbit. If orbit changes are needed to accommodate servicing, minimum accelerations must be used to permit the lightest possible telescope structures. This will, in turn, require the acceptance of extended transit times to and from servicing locations.

- Each of the three stages of EST will be built to make significant scientific contributions well beyond those of its predecessors.

- There will be a single organization responsible for EST design, construction, and operations (although operational control can change between stages: e.g., to STScI or a similar institution to task and control observations after launch).

The EST is only a design concept at this time, so detailed design of the supporting infrastructure is not yet possible. However, based on the objectives and assumptions stated elsewhere in this paper, a few high order conclusions can be reached, and then used as a foundation for more detailed analyses in parallel with the EST design process. In particular, two issues will be addressed here: location of the assembly process, and identification of a set of space vehicles upon which the servicing vehicle can be based and some thoughts on whether it must be manned or can be unmanned.

Construction and deployment of the EST is likely to occur on an approximately matching time schedule with buildup of a manned infrastructure in the lunar vicinity (taken to mean anywhere in space between geosynchronous altitude and lunar orbit). Therefore, it would be logical for these two programs to coordinate their activities, both technologically and operationally. This coordination will be assumed. In fact, the existence of at least most of the infrastructure for a large manned program may be a political/economic prerequisite for the astrophysics infrastructure.

\subsubsection{Assembly Location}

Given that the EST will be located in a SEL2 halo orbit, there will be three basic orbit classes available for assembly: Low Earth Orbit (including both LEO and GEO); the lunar vicinity in the neighborhood of the Earth-Moon libration points EML1, 2 (or a Distant Retrograde Orbit (DRO)) and the neighborhoods of the Sun-Earth libration points, SEL1, 2. With care, a significant portion of the assembly could also be conducted enroute to SEL2, but that will remain for later evaluation. 
- LEO or GEO. Starting from the Earth's surface, these are the easiest orbits to achieve, but for that very reason they require the highest energy addition to achieve the target SEL2 operational orbit for EST. This will require an orbit transfer acceleration high enough that it is likely to violate the structural requirements of the telescope; a fully assembled space telescope will almost certainly place severe restrictions on the acceptable magnitude and smoothness of acceleration, although acceptable envelopes need to be determined. For this and other reasons, both Low and Geostationary Earth Orbits should be eliminated as assembly or servicing locations for EST, even though the latency time for command and control of the operation (from the planetary surface) is lowest.

- Lunar Vicinity. Effectively, this location refers to one of the lunar libration points, EML1 (on the lunar side facing the Earth) or EML2 (opposite the Earth), or a DRO: the actual selection is likely to depend upon the specific human exploration architecture selected by NASA's Human Exploration and Operations Mission Directorate (HEOMD). When a transfer is appropriately timed, all of these orbital locations have a very low injection velocity (tens of meters per second) required for transit from and to SEL2, coupled with latency times of seconds, an operationally acceptable value. Moreover, facilities at any of the three locations can be used to support a human program of in-depth exploration of the moon, asteroid explorations, or manned Mars missions.

- Sun-Earth Libration Points. SEL2 does have the attraction as an assembly point of being the final operational orbit, but its distance from Earth is generally considered too great $\left(\sim 1.5 \times 10^{6} \mathrm{~km}\right)$ for near-future direct manned support (given the extended travel time entailed, most notably under emergency conditions, such as Apollo 13) or for the long latency time that telerobotic controls would encounter. Moreover, the SEL2 environment is reasonably benign (radiation, thermal, particulate, etc.), which is one of the reasons the location has been selected for EST and several other astronomical observatories. SEL2 can be eliminated as an assembly and servicing site primarily due to technological limitations, however, the logical solution would be to assemble the EST at EML2, transport it (at low acceleration) to SEL2 for its early operations, and then conduct maintenance and repair at either location as most appropriate. Note, however, that a final determination will depend upon the results of trades involving time enroute following assembly and maximum allowable acceleration, based upon the inherent strength of the telescope structure and the shape retention of the mirrors (if they are severely lightweighted).

\subsubsection{Assembly and Service Platforms}

Although it is possible for large space telescopes to be designed to assemble themselves in their operational orbits without further outside assistance, once the need for service, repair, or upgrade arises, one or more auxiliary service vehicles will be essential, if only to carry parts, tools, and expendables to the telescope. The International Space Station (ISS) provides an example of a very large platform that could be used to service other space vehicles but, since EST will not be serviceable in LEO, the ISS cannot be considered for other than experimental demonstrations (see below) or as a depot for parts to be carried to a higher servicing orbit. Thus, there are three classes of service platforms, which may be roughly distinguished from each other on a size basis: Macro, Mini, and Micro, each of which has a distinct role to play in establishing and maintaining EST.

- $\quad$ Macro Service Platforms. While the ISS is the dominant member of this class, most proposed Macro Platforms, while large, are much smaller than the ISS, and are designed for and capable of operating in deep space, either unmanned or manned, carrying a crew of up to four. The best known example is probably the Orion, which does have significant limitations for a service role (for example, EVA requires depressurization of the entire vehicle with the entire crew in space suits). Another example (unmanned) is presented by a Lockheed/Thales Alenia designer (designated the

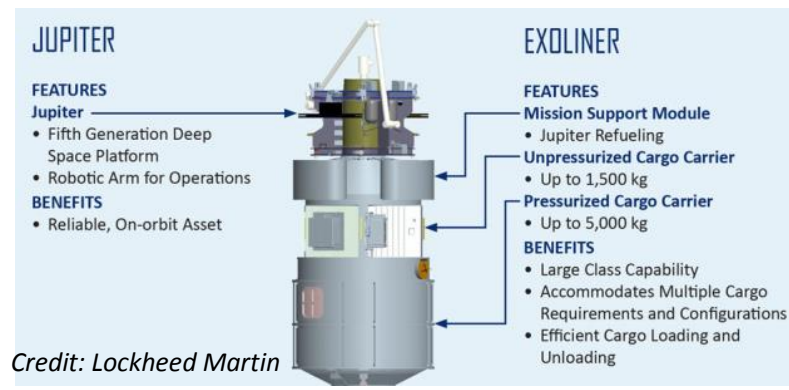

Figure 7: Jupiter ExoLiner Servicing Concept Jupiter - Exoliner ${ }^{5,6}$ ) for cargo deliveries to the ISS and beyond (to the Moon or Mars, for example) as discussed in the citations and shown in the Figure 7. There are also a number of similarly sized spacecraft 
available through the Goddard SFC Rapid Spacecraft Development Office (RSDO) that could, with varying levels of effort, form the basis for a large service platform. These would generally be used for major maintenance missions, refueling, etc. (many of which have been studied in detail), would require a dedicated launch, and, in general, are only designed for a single use.

At a larger size, there are several concepts for modest deep space habitats (DSH), that could be located at EML1 or EML2 and inhabited (possibly permanently) as needed to support lunar exploration. Each DSH could carry a crew of around four personnel who could also be involved with the assembly and maintenance of EST when needed. Although the nominal servicing mission would be accomplished robotically, human presence in the immediate vicinity would provide a potentially critical EVA backup capability.

- Mini Service Platforms. In a smaller size (perhaps $20 \%$ the length of the Jupiter Exoliner vehicle), a purely automated service platform (herein known as MiniServ) could be used to service a wide range of spacecraft in cislunar and deeper space. A MiniServ based at one of the libration points of the Sun-Earth-Moon system, could move to needed locations with minimal propellant expenditure, albeit over long periods of time. These platforms could perform a limited but useful range of maintenance functions, including parts replacement, fluids replenishment, surface recoating, and preparation of scientific spacecraft for servicing (e.g., imagery to determine specific servicing needs before larger vehicles arrive). They would be modular, permanently based in space, replenished themselves from larger vehicles performing other missions when space and time permit, and propelled by systems that could be switched out as necessary for specific missions, e.g., from pre-installed electrical to chemical propulsion for a truly urgent repair. If a DSH is available at one of the lunar libration points, the MiniServ(s) could be based at that location (perhaps permanently docked when not in use) and could be used for a variety of other missions in the DSH vicinity, including maintenance of the "mother ship" and a safety backup during crew transfers or other EVAs. Given the minimal propulsion requirements, the MiniServ could also transit from one lunar libration point to another (or to a DRO) for lunar science purposes.

- $\quad$ Micro Service Platforms. The last and smallest servicer platform, the Micro, is well represented by Spheres, a $25-30 \mathrm{~cm}$, roughly spherical vehicle that has been used inside the ISS over several years to test and demonstrate maneuvering algorithms using inert gas propulsion for operation, as shown in Figure 8. Such a system, adopted for use in vacuum conditions, could be used for auxiliary purposes, such as delivering or retrieving individual small parts or tools, imagery in areas not accessible to crew or larger robotic equipment, or other, similar missions. This, of course, would have the benefit of keeping the science system functioning at (close to) nominal design conditions or, alternatively, significantly reduce the cost of repair, while enabling inexpensive upgrades of some functions.

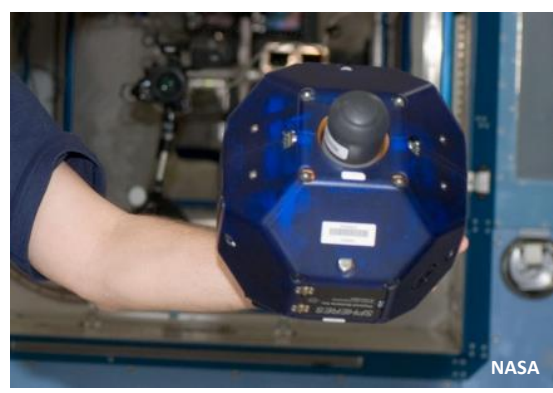

Figure 8: SPHERES Module

\subsubsection{Nominal Assembly/Servicing Concept of Operations for EST}

Based upon the preceding discussions, the following is suggested as a baseline concept of operations for the Evolvable Space Telescope:

- Technology Validations. A limited number of experimental demonstrations can be conducted on the ISS to provide stakeholders and decision makers with the required confidence in the concepts and technologies of EST to enable acquisition decisions for the system. One such experiment is briefly described in Section 4.4.

- Initial Assembly. Stage 1 of the EST would be launched directly from the surface to a DSH in the lunar vicinity $^{\dagger}$ and assembled robotically but with at-hand human supervisors, and possibly a MiniServ for assistance as needed. The completed Stage 1 vehicle would then transfer to its operational location at SEL2.

\footnotetext{
${ }^{\dagger}$ The basic argument applies to both lunar libration points (EML1 and EML2) and to a Distant Retrograde Orbit (DRO) with no compelling argument for deselection of any of them.
} 
- Subsequent Assembly. If the in-space infrastructure for Human Exploration and/or other missions has been sufficiently developed by the time, Stages 2 and 3 could be assembled at the operational (SEL2) location of the original vehicle, preferably with the assistance of an accompanying on-site MiniServ that would actually conduct most of the operation itself. Human presence would not be employed, nor would the Stage 1 EST be returned to the Lunar Vicinity base for upgrading, given the long period of withdrawal from service and the risks to the vehicle that that would entail.

- Telescope Servicing and Upgrading. These operations would be conducted in the EST operational orbit at SEL2. The preferred system to be employed in this servicing would be a MiniServ, and the EST would be designed with this desire in mind, but a larger servicing vehicle might be needed in some cases. Later in the system lifetime, a manned vehicle could be used (although this has contamination issues as well as cost/risk problems that make its implementation questionable in the lack of compelling reasons).

\subsubsection{Concept Validation in Low Earth Orbit (LEO)}

As noted above, assembly and servicing of an EST- class space telescope in LEO is not an effective approach to the support infrastructure. However, given the current and indefinite availability of the International Space Station (ISS), there are important experiments and demonstrations that can be performed in LEO to provide increased confidence in the design and technology for EST and for its supporting infrastructure. In particular, a concept known as MoDEST (Modular Demonstration of an Evolvable $\underline{\text { Space }}$ Telescope), discussed in Section 4.4, provides a concept for an orbital demonstration of several critical features of EST and of its required supporting infrastructure. It is an example of a possible near term demonstration in support of EST or other concepts for the next large observatory.

It is noteworthy that some aspects of a MiniServ system or other MicroService Platforms could also be tested using MoDEST and the current CanadaArm2 telerobotic system on the ISS. In particular, a prototype MiniServ manipulator/tool module could be loosely held by the end of the arm using a tether, moved by the arm into the proximity of a serviceable object, and then allowed to perform autonomous proximity operations near that object. The operations would be closely monitored, and the tether would provide a means of safely aborting them by pulling the module back should a malfunction be indicated.

\subsection{Modular Demonstration of an Evolvable Space Telescope}

The Modular Demonstration of an Evolvable Space Telescope or MoDEST is an early opportunity to mitigate risks and lower costs of technologies and processes needed for remote autonomous large telescope construction. MoDEST demonstrates: 1) An Evolvable Space Telescope (EST) enabling large (>12 m) HDST-like space telescopes with existing launch systems, 2) An affordable path to large apertures that are grown in stages that alleviates both peak cost per year and overall program cost growth issues, 3) On-orbit assembly of a precision telescope, and 4) Active correction of phase and mirror shape.

Working off the International Space Station (ISS) platform, MoDEST will provide an excellent near-earth opportunity to test autonomous and robotic assembly techniques as well as advanced adaptive optics technologies critical for future large telescopes.

MODEST (Figure 9) is proposed to be delivered to the International Space Station using standard Express Logistics Carriers (ELCs) and can mounted to one of a variety of ISS pallets. Post-assembly value includes space, ground, and environmental studies, a testbed for new instruments, and a tool for student exploration of space. This demonstration program for next generation mirror technology provides significant risk reduction and demonstrates the technology in a six-mirror phased telescope. Other key features of the demonstration include the use of an active primary optical surface with wavefront feedback control that allows on-orbit optimization and demonstration of precise surface control to meet optical system wavefront and stability requirements. 


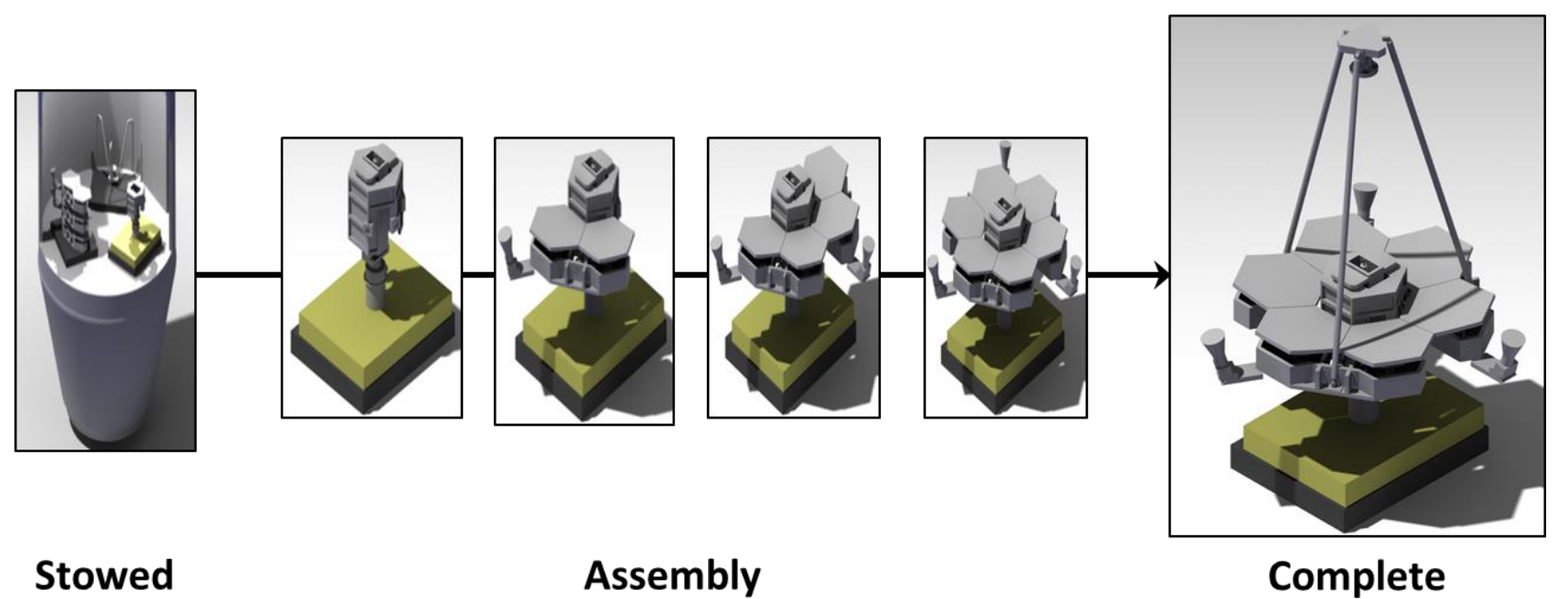

Figure 9: MoDEST ISS Testbed evolution from stowed through assembly, to final configuration

The concept of assembly is in formulation phase and envisions a SpaceX Falcon 9 booster delivering the unassembled elements of the MoDEST telescope to the ISS on a cost effective Dragon capsule. After the capsule docks the robotic arm will retrieve the MoDEST elements placing them into a transport cart which will move them to the standard mission platform for assembly. Once at the assembly area several options are available for the placement of the MoDEST elements onto the platform. The ISS host robotic arm is a candidate for both placement and assembly of the telescope components. Astronauts may play a role in the initial placement as well as observation of the MoDEST assembly processes as they progress. One key area that MoDEST will prove out is the utility of space-based Modal Image Optimization or MIO. With this approach adaptive optics can be assembled in space and algorithmically corrected for optimal optical precision. MIO optimizes deformable mirror segment phase and shape, and provides jitter control. Some benefits of MIO include:

- Minimizes mirror cost, ground testing, and risk as mirrors are tuned on orbit

- Eliminates need for a wavefront sensor; no extra optics, sensor and electronics to perform wavefront sensing

- Same algorithm/software used to phase multiple segments

- Better correction of the wavefront error induced within a single segment plus overall improvement of the wavefront error and phase diversity encountered when aligning multiple segments than is achieved with current techniques

MODEST will also be used to evaluate advances in lightweight mirror and metering structure materials such as Silicon Carbide (SiC) or Carbon Fiber Reinforced Polymer (CFRP) that have excellent mechanical and thermal properties, e.g. high stiffness, high modulus, high thermal conductivity, and low thermal expansion. It has been demonstrated that mirrors built from these materials can be rapidly replicated in a highly cost effective manner, making these materials excellent candidates for a low cost, high performance OTA.

A successful MoDEST program will help ensure that future large astrophysics systems are affordable and that exciting emerging missions are achievable.

\section{ROTATING SYNTHETIC APERTURE}

Raytheon Space and Airborne Systems initiated and began development of the concept of a rectangular Rotating Synthetic Aperture (RSA) telescope for non-astronomical applications over 20 years ago. The EST team has partnered 
with Raytheon to explore RSA as an astronomical telescope. It could offer astrophysics significant cost and complexity savings, potentially greater pointing and thermal stability, and higher spatial resolution for an equivalent cost. RSA is also relatively mature. The U.S. Government has invested nearly a third of a billion dollars in the development of technologies relevant to large RSA systems. It is likely that these technologies and concepts can be leveraged by the astrophysics community to yield the highest performing, lowest cost, large aperture astrophysics mission.

To be useful for astrophysics, aspects of RSA need to be analyzed in an astronomical context. Because of the rectangular aperture there is a trade between aspect ratio of the rectangular mirror, resolution and collecting area. This is illustrated in Table 2 were four examples of performance parameters for various aspect ratios are shown and compared to a 12-meter filled aperture similar to that discussed in the AURA report ${ }^{1}$. The RSA Integration Time Factor is the multiplicative factor needed to achieve the same signal-to-noise relative to the 12-meter filled aperture example.

Table 2 RSA Parameters and Performance Comparison

\begin{tabular}{|c|c|c|c|c|c|c|c|}
\hline \multicolumn{5}{|c|}{ Rotating Synthetic Aperture Parameters } & \multicolumn{2}{|c|}{$\begin{array}{c}\text { 12-meter Aperture } \\
\text { Comparison }\end{array}$} & \multirow{2}{*}{$\begin{array}{l}\text { Diameter of a } \\
\text { filled aperture } \\
\text { with equivalen } \\
\text { light gathering } \\
\text { capability (m) }\end{array}$} \\
\hline $\begin{array}{c}\text { Mirror } \\
\text { Aspect } \\
\text { Ratio }\end{array}$ & $\begin{array}{l}\text { Mirror } \\
\text { Length } \\
\text { (m) }\end{array}$ & $\begin{array}{c}\text { Mirror } \\
\text { Width } \\
(\mathbf{m})\end{array}$ & $\begin{array}{l}\text { Mirror } \\
\text { area } \\
\left(\mathbf{m}^{2}\right)\end{array}$ & $\begin{array}{c}\text { Angular Res @ } \\
\text { 500nm (arcsec) } \\
\text { (linear synthesis) }\end{array}$ & $\begin{array}{l}\text { RSA Fill } \\
\text { Factor }\end{array}$ & $\begin{array}{c}\text { RSA } \\
\text { Integration } \\
\text { Time factor }\end{array}$ & \\
\hline $4: 1$ & 16 & 4 & 64 & 0.00786 & $56.6 \%$ & 3.12 & 9.03 \\
\hline $6: 1$ & 18 & 3 & 54 & 0.00699 & $47.7 \%$ & 4.39 & 8.29 \\
\hline $8: 1$ & 20 & 2.5 & 50 & 0.00629 & $44.2 \%$ & 5.12 & 7.98 \\
\hline $9: 1$ & 32 & 3.5 & 113 & 0.00394 & $100 \%$ & 1 & 12 \\
\hline
\end{tabular}

The analysis of RSA for astrophysics is in its earliest stages. For the analysis we are initially assuming a launch with an existing launch vehicle and a 5-meter fairing, but later in the study we will examine launching RSA with the Space Launch System (SLS) launch vehicle and its significantly larger fairing and greater lift capacity.

We have identified a few critical attributes that are being addressed in detail. Note that these are not independent studies; all are interleaved and feed the set of system requirements:

- Collection Area vs. Resolution - Define the optimal range to address top priority astrophysics science goals.

- Aperture Aspect Ratio - Quantify the impact of non-isotropic PSF individual frames and synthesized image quality for astronomical concepts of operation and mission performance, starlight suppression for exoplanet detection and characterization (coronagraph/starshade), etc.

- Spin vs. Multi-orientation - Optimize operations for top priority astrophysics science goals

- Science Accommodation Requirements - Stability (thermal and vibrational), stray light rejection, optical telescope design (f-number, wavelength range/coatings, etc.), and detector consideration (e.g. square vs rectangular pixels, etc.)

Preliminary results from the analyses indicate that achieving an astrophysically valuable collecting area and resolution combination is relatively straight forward; while starlight suppression systems (coronagraphs, visible nullers, and starshades) appear to perform as well with RSA as they do with filled circular apertures. There appears to be no show stoppers or performance reducers for exoplanet science. Image quality performance analysis for astrophysics has just been initiated and will explore a wide range of spin rates for the telescope. Pointing and vibrational analyses are yet to begin, but no show stoppers are expected and telescope optical performance and parameters and detector considerations are not expected to present issues.

The early results from our analyses clearly indicate that RSA is a viable alternative telescope architecture that should be included in future trade analyses for the next large astrophysical observatory. 


\section{SUMMARY}

In this paper we have discussed the analyses we have conducted of two alternative architectures for future large astronomical observatories. A primary goal of this effort is to demonstrate to NASA and the science community the absolute need to explore alternate architectures to mitigate costs, adapt to new technologies, and better integrate into future in-space assembly and servicing. The space observatory environment has changed substantially over the past decades: both positively, such as advanced mirror and wavefront control technologies and a growing in-space operations capability, and negatively, in very reduced budgets. The alternate architecture approaches discussed in this paper are very viable and address all the future science goals. To implement, they will require a culture change, but we believe we should not constrain ourselves to a future of rebuilding our grandfather's space telescope.

The EST concept focuses upon how to build a large space telescope in a flat science budget era by mitigating big cost peaks, and by encouraging and embracing the development of in-space assembly and servicing infrastructure. EST starts with a modest size off-axis telescope (equivalent to a $\sim 7$-meter filled aperture telescope) that is launched as a fully functional telescope with instruments (EST Stage 1) performing first rank science. After the passage of time ( $\sim 5$ years) an augmentation mission is sent to the observatory with additional mirror segments, instruments, and other needed hardware to grow it in space to a $\sim 12$-meter filled aperture observatory (EST Stage 2). Future augmentations would again increase its size and add new instruments and support hardware to create a $\sim 20$-meter filled aperture observatory (EST Stage 3). After EST Stage 3 additional augmentations are also possible either to maintain or upgrade the 20-meter telescope for decades or to grow it to even larger sizes with added mirror elements.

The RSA concept offers a very different alternate architecture. The concept was developed for non-astronomical applications and has had a substantial amount of technical investment. Since the technology is relatively mature, our focus has been on analyzing its use for astrophysics, rather than developing a technical solution. RSA is a rectangular aperture telescope that rotates to fill the UV-plane. It offers astrophysics a lower cost, greater resolution, better stability, approach that seems to meet the starlight suppression needs for astrophysics. An assessment of the image quality expected from RSA is in progress

Again, we encourage NASA and the astrophysics community not to be constrained to only traditional space telescope approaches, but rather to fully explore and evaluate alternate architectures for these space telescopes. The technologies that enable these different approaches are either mature or maturing rapidly, so the risk levels are very manageable. They do, however, require a culture change. These alternate approaches can offer cost savings and performance enhancements over traditional methods and can enable a more capable astrophysical observatory earlier in time than the traditional approach.

\section{ACKNOWLEDGEMENTS}

The authors would like to acknowledge strong support and internal funding from Northrop Grumman Aerospace Systems and very helpful comments, suggestions, and criticisms from a variety of people, including Jonathan Arenberg, Suzanne Casement, Alberto Conti, Michael Triller, Amber Bauermeister, Mark Folkman, Keith Leavitt, Mitchell Haeri, Neil Malone, Susan B. Spencer, Douglas W. Wolfe, Marc Postman, Ken Sembach, Wes Traub, and Harley Thronson. 


\section{REFERENCES}

[1] Dalcanton, J., et.al, "From Cosmic Birth to Living Earths: The Future of UVOIR Astronomy," Association of Universities for Research in Astronomy (AURA), 2015

[2] Stahl, H. P., et al; "Update to single-variable parametric cost models for space telescopes", Opt. Eng. 52(9) 091805 doi: 10.1117/1.OE.52.9.091805

[3] Polidan R. S., Breckinridge J. B., Lillie, C. F., MacEwen, H. A., Flannery, M. R., Dailey, D. R., “An evolvable space telescope for future astronomical missions", Proc. SPIE 9143-19, Montréal, Quebec, Canada, 2014

[4] Underwood, C., Pellegrino, S., ...J. Breckinridge...et al "Autonomous Assembly of a Reconfigurable Space Telescope (AAReST) - a cubesat based technology demonstrator”, 27th annual AIAA/USU conference on small satellites, (2013)

[5] Lam, W. T., and Chipman, R., "Balancing polarization aberrations in crossed fold mirrors", Appl. Opt. 54, 3236$3245,(2015)$

[6] Clark, N., and Breckinridge, J., "Polarization compensation of Fresnel aberrations in telescopes"., SPIE 8146 \# 814600, (2011)

[7] Wetherell, W. B. , and M. P. Rimmer, "General analysis of aplanatic Cassegrain, Gregorian, and Schwarzschild telescopes,", Appl. Opt. 11, 2817-2832 (1972)

[8] Budano, A., Flora, F. and Mezi, L. “Analytical design method for a modified Schwarzschild optics,"Appl. Opt. 45, 4254-4262 (2006).

[9] Breckinridge, J. B., Lam, Wai Sze T. and Chipman, Russell A. "Polarization Aberrations in Astronomical Telescopes: The Point Spread Function", Publications of the Astronomical Society of the Pacific (PASP), 127:445468 (2015)

[10] Breckinridge, J. B., "Polarization properties of a grating spectrograph" Applied Optics, 10, 286-294, (1971)

[11] J. B. Breckinridge, J. B., and Oppenheimer, B., "Polarization Effects in Reflecting Coronagraphs for White Light Applications in Astronomy", Astrophysical Journal, 600, pp 1091 - 1098. (2004)

[12] National Aeronautics and Space Administration, Goddard Space Flight Center, "On-Orbit Satellite Servicing Study Project Report", NASA GSFC SSCP-RPT-000144, October 2010: Available at http://ssco.gsfc.nasa.gov/images/NASA_Satellite\%20Servicing_Project_Report_0511.pdf

[13] Foust, J., "Lockheed Pitches Reusable Tug for Space Station Resupply”, Space News, 13 March 2015; http://spacenews.com/lockheed-martin-pitches-reusable-tug-for-space-station-resupply

[14] Morring, Frank Jr., “Jupiter' Space Tug Could Deliver Cargo To The Moon: Lockheed, MDA, Thales Alenia team on ISS and deep-space cargo carrier", Aviation Week \& Space Technology, March 12, 2015 http://aviationweek.com/space/jupiter-space-tug-could-deliver-cargo-moon 